\title{
Evaluation of Different Genomic Alterations in Patients of Thyroid Cancer
}

\author{
Muhammad Ameen* \\ Arid Agricultural University, Pakistan \\ *Corresponding author: Muhammad Ameen, Pakistan
}

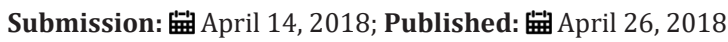

\begin{abstract}
The polymerase chain reaction (PCR) is enzymatic amplification of specific regions of DNA while the inverse polymerase chain reaction (IPCR) is used for obtaining flanking regions of unknown sequences without utilizing cloning techniques. The template for the reverse primers is a restriction fragment that has been ligated upon it to form a circle. The base changes and sequences of DNA can be amplified by using labeled substrates in polymerase chain reaction and non-denaturing polyacrylamide gels, the electrophoretic mobility of single-stranded nucleic acid depends not only on its size but also on its sequence. Mobility shifts have used to determine single base changes up to 200-base fragments. A comprehensive statistical analysis called $\chi 2$ test has done in order to evaluate the sample of 200 individuals of both experimental group and control group. The blood tests reports with detailed history was collected with the help of clinicians in NORI and entered in the Performa. The current study will determine various types of thyroid cancer with respect to genetic alterations in aforementioned populations.
\end{abstract}

\section{Introduction}

Hundahl (1998) have demonstrated that thyroid tumor the most prominent endocrine tumors has documented into four types, first one follicular epithelial cell determined papillary thyroid cancer which represents more or less $80 \%$, second type is follicular thyroid cancer represents $15 \%$, third type is anaplastic thyroid cancer represents $2 \%$ and para-follicular C-cell inferred medullary thyroid cancer.

Samuels et al. [1] have determined that V600E BRAF mutation usually occurs in PTC which is typical endocrine malignancy. In current years, this mutation has gotten impressive consideration due to its use in administration of papillary thyroid cancer. In papillary thyroid cancer BRAF mutation is firmly connected with additional thyroidal expansion, LNM, propelled cancer stages, repetition of the disease and with death rate of the patient. BRAF mutation was identified to responsible for various molecular imbalances increase expression of genes that promote tumor and suppression of genes that control iodine in thyroid bringing about hindrance of radioiodine voracity as a result causes disappointment in cure of papillary thyroid cancer by iodine radiation.

Handkiewicz et al. [2] have reported that BRAF is much of the time connected with aberrant methylation of a few tumor silencer genes, for example TIMP, DAPK and RARb2. In vitro studies have exhibited that expanded articulation of matrix metalloproteinase three, nine and thirteen, vascular endothelial growth factor and stimulation of nuclear transcription factor.
Polymerase chain reaction in order to determine the flanking regions of unknown sequences of DNA will be conducted in this innovative research paper. Evaluation of various publications has shown that aberrations in different genes are responsible for thyroid carcinoma in different studied populations. Such innovative work regarding polymerase chain reaction in patients of thyroid cancer is lacking in Pakistan. This study is designed to evaluate possible contribution of genetic alteration in patients with thyroid cancer in aforementioned population.

The main objective of this research strategy is to determine the extent of BRAF and PIK3CA genes mutations through polymerase chain reaction.

\section{Materials and Methods}

The innovative research strategy was carried out at Nuclear Medicine, Oncology and Radiotherapy Institute (NORI) between patients of thyroid cancer versus normal control individuals. A sample of population constituting 200 individuals was categorized into two major groups, one is experimental group and other is control group. A population base case versus control study was conducted in 150 patients of thyroid cancer matched with 50 control healthy individuals. The patients having thyroid cancer were diagnosed through Physical examination, clinical examination, Thyroid Scan, and Ultrasound reports. 
It has incorporated two genes i.e. BRAF and PIK3CA for mutational analysis. BRAF is the most common genetic alteration in thyroid cancer that activates MAPK pathway leading to oncogenic cell proliferation. PIK3CA gene part of phosphatidylinsitol 3-kinase (PI3K) contributes to a typical cell development and cellular makeover in different types of cancer including thyroid cancer. The purpose of this study was to determine extent of involvement of alterations of these genes in thyroid cancer in Pakistani population. Hypothesis has tested that genetic variation in BRAF and PIK3CA plays role in etiology of thyroid cancer. Phenol chloroform method was used for DNA extraction and spectrophotometry. It was done for the quantification of DNA.

\section{Collections of samples}

Blood samples of cancer Patients were collected at Nuclear Medicine, Oncology and Radiotherapy Institute (NORI), Pakistan. Patients were recognized by oncologist of the (NORI) hospital. Disease free and healthy individuals were considered as Control group while patients of goiter constituted experimental group. Patients were not forced for sampling, on the interest of patients their blood were collected. Blood samples of $2 \mathrm{ml}$ were collected in Annexure-I:
EDTA-containing tubes. Samples were stored at -20C until further use.

\section{Setting and study area}

The present study was carried out in Nuclear Medicine, Oncology and Radiotherapy Institute (NORI).

\section{Study population}

A comprehensive survey of 200 individuals will be carried out.

\section{Inclusion criteria}

All patients having thyroid tumor will be documented in this study.

\section{Exclusion criteria}

Patients having no thyroid cancer and tumor will be excluded

Study group: The study population was based on the prevalence of BRAF and PIK3CA mutations that were observed in the patients of thyroid cancer. Therefore, the whole population sample constituting the patients of thyroid cancer has based on its types.

Annexure-II:

\begin{tabular}{|c|c|c|c|}
\hline \multicolumn{2}{|l|}{} & If Yes, Duration/Date & Remarks (if any) \\
\hline H/O Goiter & Yes/No & & \\
\hline Type & Papillary & Follicular & Follicular variant \\
\hline Physical Examination & & & \\
\hline Thyroid scan: & & & \\
\hline Thyroid Ultrasound & & & \\
& & & \\
\hline
\end{tabular}


Data collection: After having informed consent (Annexure-I) from patients, the data along with detailed history was collected with the help of clinicians in NORI and entered in the Performa (Annexure-II).

\section{DNA extraction}

Leukocytes were used to extract DNA of both cancer patients as well as normal individuals. Agarose gel (1\%) is used for the process of Electrophoresis. DNA bands were stained by Ethidium bromide in UV transilluminator.

\section{General solutions}

In this study some solutions are used on daily basis.

\section{X Tris, boric acid, EDTA (TBE)}

$539 \mathrm{~g}$ of Tris, $274 \mathrm{~g}$ boric acid and 45.9g of disodium EDTA were dissolved in $\mathrm{dH}_{2} \mathrm{O}$ and volume $10 \mathrm{~L}$ was made.1L was diluted to $1 \mathrm{x}$ concentration before use in agarose gel electrophoresis and keeps the solution at room temperature.

\section{Polymerase chain reaction (PCR)}

Primers designing for BRAF and PIK3CA: Genetic alteration in exon 16 of BRAF and exon 9 of PIK3CA were examined through gene Primer 3 input software versions. Primers were also checked by national centre for biotechnology information (Table 1).

Table 1:

\begin{tabular}{|c|c|c|c|}
\hline BRAF Primers & Sequence & Product Mass & 224 \\
\hline Forward & TCATAATGCTTGCTCTGATAGGA & 224 & 58 \\
\hline Reverse & GGCCAAAAATTTAATCAGTGGA & Product mass & 205 \\
\hline PIK3CA primers & Sequence & Temperature & 205 \\
\hline Forward & ATCATCTGTGAATCCAGA & 58 \\
\hline Reverse & TTAGCACTTACCTGTGAC & 58 \\
\hline
\end{tabular}

\section{Designing of primers for BRAF gene}

Optimization of primers: It was necessary to optimize BRAF and PIK3CA genes primers for their precise annealing temperature. The primers were run on polyacrylamide gel electrophoresis to find any non-precise bands.

Thyroid tumor samples and controls amplification: Polymerase chain reaction was performed by 10ul PCR sample mixture comprising $5 \mathrm{ul}$ master mix, $1 \mathrm{ul}$ primer, $2 \mathrm{ul}$ PCR water and 2ul PCR water and 2ul of DNA that was prepared in PCR workstation to minimize any cross contamination. Reaction mixture was placed in gene AMP PCR system 9700 and verity 96 well thermal cycler were utilized. Amplification was done for all diseased and normal DNA samples for definite genes with specific genes with specific exon and for precise primers. Negative controls were also used in order to rule out any contamination or non-specificity.

Making of sample for SSCP: For determination of genetic changes SSCP is one of the cheapest and specific techniques. Little measure of enhanced items was moved into little tubes. Same quantity of denaturant $(\mathrm{NaOH})$ was also put in these tubes and short was given to samples for blending. A solution of 5 ul enhanced product of PCR and $5 \mathrm{ul}$ of $\mathrm{NaOH}$ was used in single strand chain polymorphism.

Samples denaturation: At temperature 95C single strand chain polymorphism samples were denatured for 10 minutes than quickly moved on ice. This warmth stun was given for breakage of hydrogen bonds between DNA helix and converted double stranded DNA into single stranded Lallas and Buller. (1998). Single stranded DNA was held by keeping it on ice for $5 \mathrm{~min}$ after this extraordinary warming at high temperature.

Making of PAGE: For making PAGE gel plates of different chemicals were used and these chemicals were used in different quantity to make $50 \mathrm{ml}$ volume. The volume of chemical are shown as below.

a) Acrylamide solution

$14 \mathrm{ml}$

b) 10X Tris base EDTA

$4.5 \mathrm{ml}$

c) Ammonium persulphate

$355 \mathrm{ul}$

d) Tetra methyl ethylene diamine

Circumstances for electrophoresis: The plates were balanced on vertical gel device as gel was polymerized. The running buffer used in electrophoresis was 1Xtris, borate EDTA. Electrophoresis was run at $130 \mathrm{~V}$ for one and half hour. The device was placed in cool environment to overwhelmed a change in temperature that may affect the movement of the samples fresh buffer was used for each turn to increase sensitivity.

Imaging and staining gel: Gel documentation system was used for imagining gel was moved to this system for capturing pictures and save those pictures for future analysis.

Variants recognition and their sequencing: Single strand chain polymorphism amplified products which showed unusual pattern were choosing for sequencing in forward direction. Samples were transported to MC lab (California) for sequencing.

\section{Statistical analysis}

Univariate and multivariate analysis were performed in order to test significant association. The p values equal or less than 0.05 . were considered to be significant.

\section{Thyroid cancer and occurrence of BRAF V 600E Mutation}

Optimizations of primers: The optimization of BRAF and PICK3 was done according to specified size of product on specified temperature in order to obtain a solitary band of specified product size a scope of temperature and reagents fixations were utilized. On 
$2 \%$ agarose gel amplified products were stacked. Electrophoresis delineates the accomplishments of polymerization as well as portrays any possibilities of plausible non specificity. Samples were to be utilized for mutation detection later it was essential to evacuate the shorts of any non-particular binding of primers. Ladder (100bp) was utilized as ruler for the estimation of product size.

\section{Results and Discussion}

The comprehensive research strategy has determined significant difference through the statistical evaluation of thyroid Table 2: $\mathrm{X}^{2}$ Calculations (Two-way Contingency) showing statistical difference between cancer patients versus normal control individuals.

\begin{tabular}{|c|c|c|c|c|c|}
\hline Number of Patients with Thyroid Cancer & Total No. & Male & Female & Control & \\
\hline \multirow{3}{*}{ Papillary } & 110 & 37 & 73 & 50 & \multirow{3}{*}{270} \\
\hline & 90 & 30 & 60 & 90 & \\
\hline & -4.44 & -1.63 & -2.82 & -17.78 & \\
\hline \multirow{3}{*}{ Follicular } & 30 & 10 & 20 & 50 & \multirow{3}{*}{110} \\
\hline & 36.67 & 12.22 & 24.44 & 36.67 & \\
\hline & -1.21 & -0.4 & -0.81 & -4.85 & \\
\hline \multirow{4}{*}{ Follicular variant } & 10 & 3 & 7 & 50 & \multirow{3}{*}{70} \\
\hline & 23.33 & 7.78 & 15.56 & 23.33 & \\
\hline & -7.62 & -2.93 & -4.71 & -30.48 & \\
\hline & 150 & 50 & 100 & 150 & 450 \\
\hline
\end{tabular}

$\mathrm{x}^{2}=79.681, \mathrm{df}=6, \mathrm{x}^{2} / \mathrm{df}=13.28, \quad \mathrm{P}\left(\mathrm{x}^{2}>79.681\right)=0.0000$

Table 3: $\mathrm{X}^{2}$ Calculations (Two-way Contingency) showing statistical difference between cancer patients of various age groups versus normal control individuals.

\begin{tabular}{|c|c|c|c|c|c|}
\hline Number of Patients with Age Groups(Years) & Total No. & Male & Female & Control & \\
\hline \multirow{3}{*}{$20-40$} & 30 & 10 & 20 & 50 & \multirow{3}{*}{110} \\
\hline & 36.67 & 9.78 & 26.89 & 36.67 & \\
\hline & -1.21 & -0.01 & -1.76 & -4.85 & \\
\hline \multirow{3}{*}{$40-60$} & 40 & 10 & 30 & 50 & \multirow{3}{*}{130} \\
\hline & 43.33 & 11.56 & 31.78 & 43.33 & \\
\hline & -0.26 & -0.21 & -0.1 & -1.03 & \\
\hline \multirow{3}{*}{$60-80$} & \multirow{3}{*}{$\begin{array}{l}80 \\
70\end{array}$} & 20 & 60 & 50 & \multirow{3}{*}{210} \\
\hline & & 18.67 & 51.33 & 70 & \\
\hline & & -0.1 & & & \\
\hline
\end{tabular}

$x^{2}=18.123, \mathrm{df}=6, \quad \mathrm{x}^{2} / \mathrm{df}=3.02, \mathrm{P}\left(\mathrm{x}^{2}>18.123\right)=0.0059$

The appropriate statistical analysis in Table 3 demonstrated that female percentage (67\%) Was grater as compared to male percentage $(33 \%)$. It showed that in Pakistani population the prevalence of thyroid cancer is higher in females than in males. This result was in consistency with other researchers Rahbari et al. [3] analyzed annually that thyroid cancer is 2.9-times less common in men than women. Jemal et al. [4] reported that the predominance of thyroid malignancy has expanded up to fifty percent since 1973, it is the 2nd common cancer in men and it is the most quickly expending disease among ladies. Sajid et al. [5] have reported that thyroid cancer has been observed more common in females, and males to females ratio was $1: 2$. cancer patients versus normal control individuals. The occurrence of thyroid cancer was maximum in patients having BRAF and PICK3 mutations comparatively to normal control individuals. Therefore, both BRAF and PIK3CA gene are the most common genetic alteration in thyroid cancer the former activates MAPK pathway leading to oncogenic cell proliferation while latter contributes to a typical cell development and cellular make over in different types of cancer including thyroid cancer. The study has justified that both BRAF and PIK3CA gene alterations have played a vital role in thyroid cancer (Table 2). 


\section{Conclusion}

The present study concluded that prevalence of thyroid cancer is dependent on the extent of both BRAF and PIK3CA gene alterations. In future comprehensive studies are warrant covering the epidemiological profile of the patients having thyroid cancer.

\section{References}

1. Samuels Y, Wang Z, Bardelli A, Silliman N, Ptak J (2004) High frequency of mutations of the PIK3CA gene inn human cancers. Science 304(5670): 554-556.

2. Handkiewicz JD, Czarniecka A, Jarzab B (2010) Molecular prognostic markers in papillary and follicular thyroid cancer: Current status and future directions. Mol Cell Endocrinol 322(1-2): 8-28.

3. Rahbari R, Zhang L, Kebebwe E (2010) Thyroid cancer gender disparity. Future Oncole 6(11): 1771-1779.
4. Jemal A, Murray T, Ward E (2005) American cancer society, cancer statistics. Cancer J Clin 55: 10-30.

5. Sajid M, Ringel MD (2010) The p13k-akt-mTOR pathway in initiation of progressive of thyroid tumors. Mol Cell Endocrinol 321(1): 20-28.

6. Kebebew MD, Weng BSJ, Juergen B, Gustavo R, Clark H, et al. (2007) The prevalence and prognostic value of BRAF mutation in thyroid cancer Anm Surg 246(3): 466-471.

7. Trovisco V, Vieeria DCL, Soares P (2004) BRAF mutation is associated with some histological types of papillary thyroid carcinoma. J Pathol 202(2): 247-251.

8. Fugazzola L, Puxeddu E, Avenia N (2006) Correlation between BRAFV600E mutation and clinio-pathologic parameters in papillary thyroid carcinoma data from a multicentric Italian and review of literature. Endocr Relat Cancer 13(2): 455-464.

9. Glettre E, Kravdal O (1994) Male and female parity and risk of thyroid cancer. Int J Cancer 58(4): 616-617.

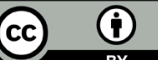

Creative Commons Attribution 4.0 International License

For possible submissions Click Here

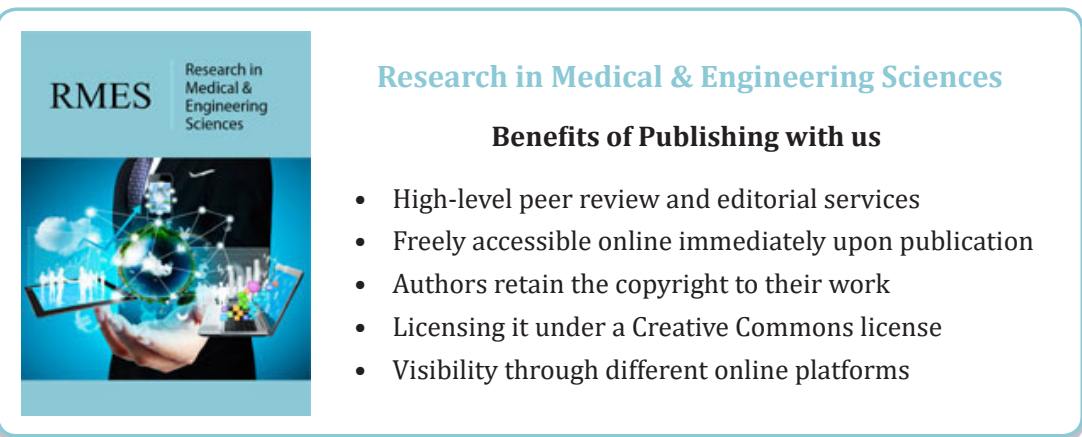

